\title{
Light absorption by an inhomogeneous semiconductor film
}

\author{
L. Baraban ${ }^{1}$, V. Lozovski ${ }^{1,2}$ \\ ${ }^{1}$ Taras Shevchenko Kyiv National University, Radiophysics Department, \\ 2/5, prospect Academician Glushkov, 03022 Kyiv, Ukraine, e-mail: laryssy@univ.kiev.ua \\ ${ }^{2}$ V. Lashkaryov Institute of Semiconductor Physics, NAS of Ukraine, \\ 45, prospect Nauky, 03028 Kyiv, Ukraine \\ Corresponding author: V. Lozovski, e-mail: lozovski@univ.kiev.ua
}

\begin{abstract}
Processes of light absorption by thin semiconductor film in the framework of local-field method are studied. The film is inhomogeneously implanted with $\mathrm{O}^{+}$ions. A distribution of implanted layer is characterized by different profiles. The effective susceptibility (response to the external field) and dissipative function of inhomogeneous in thickness semiconductor film were calculated. The absorption spectra are numerically calculated as a function of the frequency and angle of incidence. It was obtain that light absorption spectra are strongly dependent on profile distributions of implanted impurities along the film thickness.
\end{abstract}

Keywords: thin film, local field, effective susceptibility, absorption, semiconductor, implanted ion.

Manuscript received 23.06.05; accepted for publication 25.10.05.

\section{Introduction}

Thin and ultra-thin films are in interest for fundamental and applied sciences. This is associated with the investigations of new materials for different areas of human activities. Nowadays the technologies allow to create the devices based on thin films. The letter can be both solid films (for example, quantum wells and superlattices [1-4]) and molecular coatings [5-6]. The devices based on ultra-thin films, for example superlattices, characterized by quantum-dimension effects, are widely used in modern electronics [7-9]. It should be also pointed on the example of using the classical properties of thin films. One of the branches of thin film science is associated with developing and producing the sensors based on the surface plasmonpolariton resonance effect $[10,11]$.

The macroscopic Maxwell equations can be applied only to the systems, where the wavelength $\lambda$ is much longer than the characteristic dimensions of the microscopic field variations $l$, and characteristic linear dimension of the particle $L$ is much longer than $l$. Otherwise, other methods should be developed for describing the electrodynamical properties. The case of electrodynamics of mesoparticles where the thickness of the transition layer near the surfaces of the particles is about $l$ and $L \sim l$ can be mentioned as an example. Transition layers are characterized by extremely inhomogeneous local fields. In the cases of thin films, the transition layers at both interfaces are about the thickness of the film. It means that the condition $L>>l$ is not carried out for the thin film, and the standard methods of macroscopical electrodynamics for these films cannot be used. The development of near-field physics gives the powerful method for calculation of electrodynamical properties of nanoobjects based on solution of the Lippmann-Schwinger equation for selfconsistent fields $[12,13]$. The main idea of the localfield method is to ascertain the relation between the local field in an arbitrary point inside the system $\vec{E}(\vec{R}, \omega)$ and external long-range field $\vec{E}^{(0)}(\vec{R}, \omega)$ that is written as

$E_{i}(\vec{R}, \omega)=L_{i j}(\vec{R}, \omega) E_{j}^{(0)}(\vec{R}, \omega)$

with the local-field factor $L_{i j}(\vec{R}, \omega)$ [12]. The method of effective susceptibility allows to calculate the local-field factor via the linear response (to the external field) function.

The theory of electrodynamical properties of ultrathin films based on the method analogous to the meanfield theory $[14,15]$ was developed earlier. The main idea of this theory was to exclude uniform field characterizing the film from all equations for the Green function. As a result, the equation for the electrodynamical Green function of ultra-thin film was obtained. The tensor of the linear response to the external field 
was also calculated. It should be emphasized that the elctrodynamics of ultra-thin films could be built using the specific boundary conditions that require the local field abrupt across the upper and bottom sides of the film (see, for example Refs [16] and [17]).

In this work, we consider the absorption of the external electromagnetic field by a thin inhomogeneous semiconductor film irradiated by an electromagnetic wave. The consideration was performed using an approach based on the solution of the LippmannSchwinger equation with regard to the local-field effects proposed in Refs [18, 19].

\section{Model}

Let us consider a thin semiconductor $n^{+}-\mathrm{GaAs}$ film with some distribution of compensating impurity. Electrons are compensated by positive ions of oxygen in the certain film area, defined by the distribution of compensating impurity. The dielectric permeability of the material is determined by both phonon and plasma of free carriers excitations. In the simplest model of the dielectric function for semiconductor, the dielectric permeability can be written in the form

$$
\varepsilon(\omega, z)=\varepsilon_{\infty}\left(1+\frac{\omega_{L}^{2}-\omega_{T}^{2}}{\omega_{T}^{2}-\omega^{2}-i \omega \gamma_{\mathrm{ph}}}-\frac{\omega_{P}^{2}}{\omega^{2}+i \omega \gamma_{\mathrm{pl}}}\right),
$$

where $\varepsilon_{\infty}$ is the high frequency dielectric permeability; $\omega_{L}, \omega_{T}, \omega_{P}$ are the longitudinal and transversal frequencies of phonons as well as the plasma frequency, respectively; $\gamma_{\mathrm{ph}}, \gamma_{\mathrm{pl}}$ are the phonons and plasma excitation decay constants. The density of electrons along the thickness of the film depends on the profile of compensating impurities $\mathrm{O}^{+}$(see. Fig. 1). It leads to dependence of the plasmon frequency $\omega_{P}$ on the z-coordinate inside the film. The shape of impurity distribution can be determined by the theory of ionic implantation by Lindhard-Scharf-Shiott [20]. According to the basic principles of this theory, implanted ions are distributed along the thickness of the film with the Gauss function

$$
n_{i}(z)=\frac{N}{\sqrt{2 \pi} \Delta R_{p}} \exp \left\{-\frac{1}{2}\left(\begin{array}{c}
z-R_{p} \\
\Delta R_{p}
\end{array}\right)^{2}\right\},
$$

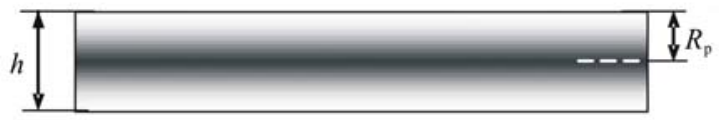

Fig. 1. Dependence of electron concentration on coordinate along the thickness of the film. The dark area corresponds to the high concentration of charge carriers. with the doze of implanted ions $N$, the depth of distribution $R_{p}$, and the dispersion of distribution $\Delta R_{p}$. The space-dependent linear response to the total (local) field is the characteristic feature of the model under consideration. In agreement with Eq. (3), the plasmon frequency is dependent on the $z$-coordinate

$$
\omega_{P}^{2}=\left(\frac{4 \pi e^{2} n(z)}{m^{*} \varepsilon_{\infty}}\right)^{1 / 2} .
$$

Consequently, the dielectric function must be the function of the $z$-coordinate. Then, the problem is to calculate the local self-consistent field inside the film with regard to both inhomogeneity of initial susceptibility of the material of the film and local-field effects caused by inhomogeneities. It is necessary to point out that study of the same problem, where consideration of the optical properties of thin semiconductor film doped with the compensating impurity was provided by using the model of the steplike distribution of compensating impurity [21]. Nevertheless this extremely simplified model of ionic implantation (see, Fig. 2a) gave suitable results for optical properties of the implanted thin film. It is clear, that in the real situation, the profile of impurity distribution has a more complicated dependence on a $\mathrm{Z}$ coordinate. In this case, the multistep-like model (Fig. 2b) could be used analogously to the one-step-like model and, of course, could give a more adequate description of the film optical properties. Consequently, the plasmon oscillations excite only on the single frequency for each step, and to describe properties of the whole film the multiplayer model should be used. This model is rather complicated. Moreover, such assumption does not correspond to a real physical situation completely as far as the so-called "tails of distribution" exist and can contribute to the general properties of a film. Then, here we shall use the recently developed approach [22] where the characteristics of the film material can continuously change along its thickness. The concentration of electrons along the film thickness is ever-changing, its plasmon frequency is everchanging, too. It leads to the dependence of the initial susceptibility (the response to a local field) both on the frequency and z-coordinate (Fig. 3).

\section{The linear response to the external field}

The effective susceptibility of a thin film is the important characteristic showing how the film responds to an external field. To understand a physical sense of the effective susceptibility, one need to consider the general solution of the Lippmann-Schwinger equation, that describes the self-consistent (local) field, formed inside a particle $[12,13]$. The equation for a thin film can be written in the form 

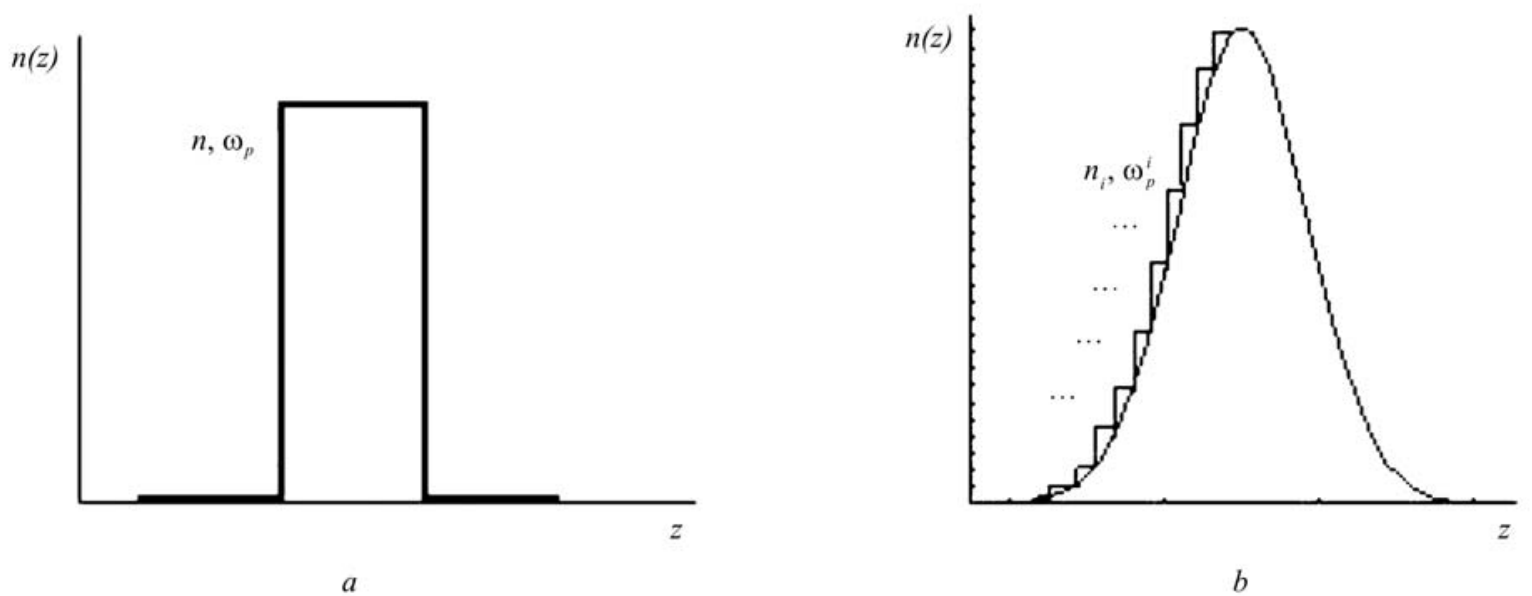

Fig. 2. Single step-like (a), multistep-like (b) and smooth Gauss-like (b) distribution of the compensating impurity.

$$
\begin{aligned}
& E_{i}(\vec{k}, z, \omega)=E_{i}^{(0)}(\vec{k}, z, \omega)- \\
& -\frac{1}{4 \pi \varepsilon_{0}} \int_{-h / 2}^{h / 2} d z^{\prime} G_{i j}\left(\vec{k}, z, z^{\prime}, \omega\right) \chi_{j l}\left(\omega, z^{\prime}\right) E_{l}\left(\vec{k}, z^{\prime}\right),
\end{aligned}
$$

with $E_{i}^{(0)}(\vec{k}, z, \omega)$ as the external longwave field, $\chi_{j l}\left(\omega, z^{\prime}\right)$ as the linear response to the local field, and $\vec{k}$ as the projection of the external field wavevector on the film plane. The solution of the Lippmann-Schwinger equation (5) can be written via an effective susceptibility (the linear response to an external film) in the form:

$$
\begin{aligned}
& E_{i}(\vec{k}, z, \omega)=E_{i}^{(0)}(\vec{k}, z, \omega)- \\
& -\frac{1}{4 \pi \varepsilon_{0}} \int_{-h / 2}^{h / 2} d z^{\prime} G_{i j}\left(\vec{k}, z, z^{\prime}, \omega\right) X_{j l}\left(\vec{k}, z^{\prime}, \omega\right) E_{l}^{(0)}\left(\vec{k}, z^{\prime}\right),
\end{aligned}
$$

where the effective susceptibility is [22]

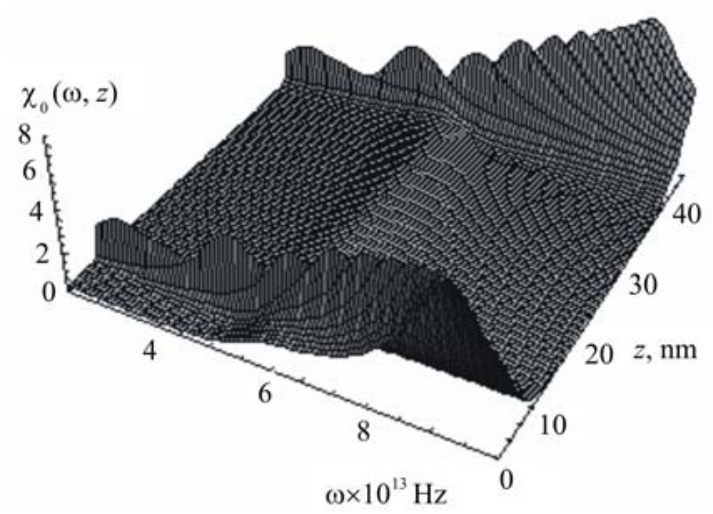

Fig. 3. The real part of the initial susceptibility as a function of the frequency and z-coordinate (along the thickness of the film) $\operatorname{Re}\left[\chi_{0}(\omega, z)\right]$. The depth and dispersion of the distribution are $R_{p}=0.5 \mathrm{~h}$ and $\Delta R_{p}=0.11 \mathrm{~h}$, respectively.

$$
\mathrm{X}_{j m}(\vec{k}, z, \omega)=\chi_{j l}(\omega, z) P_{l m}(\vec{k}, z, \omega)^{-1},
$$

with

$$
\begin{aligned}
& P_{l m}(\vec{k}, z, \omega)=\left[\delta_{m l}+\right. \\
& \left.+\frac{1}{4 \pi \varepsilon_{0}} \int_{-h / 2}^{h / 2} d z^{\prime \prime} G_{m j}\left(\vec{k}, z^{\prime}, z^{\prime \prime}\right) \chi_{j l}(\omega, z) e^{i q\left(z-z^{\prime}\right)}\right]^{-1}
\end{aligned}
$$

local-field correction function. In this expression, $q$ is the $z$-component of the external field wavevector. Then, the effective susceptibility (7) connects the longwave external field with the local field $E_{i}(\vec{k}, z, \omega)$ that is quickly changed at short-range distances. As can be seen from Eq. (8), the proposed approach can be easily used for calculation of the effective susceptibility of an inhomogeneous film, for example, for the film with inhomogeneous distribution of compensating impurities along its thickness. One should note that different widths of the implanted ion distribution profile mean the different relationship between the contributions of the polariton and plasmon subsystems into the formation of electrical response to a local field. As can be easily seen from Eq. (7), the same behavior can be also observed for the effective susceptibility.

\section{Light absorption by thin semiconductor film}

The self-consistent approach allows to describe the specific features of the absorption of an electromagnetic wave energy by a thin film. The absorbed energy of electromagnetic wave in a unit film area per unit time can be written:

$$
Q=\left\langle\left(\vec{J}+\vec{J}^{*}\right)\left(\vec{E}+\vec{E}^{*}\right)\right.
$$


where $\vec{J}(\vec{R})$ is the local current in the film caused by the local field $\vec{E}(\vec{R}) ; \overline{(\ldots)}$ and $\quad \ldots$. are the time averaging over the period (much larger than the period of the electromagnetic field oscillation), and averaging over the film volume, respectively. Local currents are related to the effective susceptibility via equation

$$
\vec{J}(\vec{R})=-i \omega \cdot \vec{X}^{(E)} \vec{E}^{(0)}(\vec{R}) .
$$

On the other side, the local field is related to the external field as

$$
\vec{E}(\vec{R})=\left(\vec{\chi}^{(E)}\right)^{-1} \vec{X}^{(E)} \vec{E}^{(0)} .
$$

Using the substitution of Eqs (10) and (11) into Eq. (9) and performing the averaging-out over time, one obtains

$$
\begin{aligned}
& Q=-i \omega\left\langle\vec{X}^{(E)}\left(\left(\vec{\chi}^{(E)}\right)^{-1} \vec{X}^{(E)}\right)^{*}-\right. \\
& \left.-\left(\vec{X}^{(E)}\right)^{*}\left(\left(\vec{\chi}^{(E)}\right)^{-1} \vec{X}^{(E)}\right)\right\rangle \vec{E}^{(0)} \vec{E}^{(0)} .
\end{aligned}
$$

Using Eq. (7) into Eq. (12), one can write for the case of isotropic initial susceptibility that energy absorbed by the inhomogeneous film can be written in the form

$$
Q=2 \omega \cdot I_{0} \frac{1}{h} \int_{-h / 2}^{h / 2} d z \operatorname{Im}(\chi(z, \omega))|\vec{P}(\vec{k}, z, \omega)|^{2} \vec{e} \cdot \vec{e}(
$$

with the unit vector of light polarization $\vec{e}$, the intensity of incident light $I_{0}=\left|E^{(0)}\right|^{2}$. Thus, the absorbed energy is controlled by the imaginary part of the initial susceptibility of the film material $\chi_{0}(\omega, z)$, and defined by the film thickness and experiment geometry.

The main contribution of local field effects is displayed by the pole part of the effective susceptibility, or as seen from Eq. (7)

$$
\begin{aligned}
& \operatorname{det}\left[\delta_{m l}+\right. \\
& \left.+\frac{1}{4 \pi \varepsilon_{0}} \int_{-h / 2}^{h / 2} d z^{\prime \prime} G_{m j}\left(\vec{k}, z^{\prime}, z^{\prime \prime}\right) \chi_{j l}(\omega, z) e^{i q\left(z-z^{\prime}\right)}\right] .
\end{aligned}
$$

In other words, the specific absorption occurs in the resonant case, when the real part of the pole part of the function $\vec{P}(\vec{k}, z, \omega)$ is equal to zero

$$
\begin{aligned}
& \operatorname{Re}\left\{\operatorname { d e t } \left[\delta_{m l}+\right.\right. \\
& \left.\left.+\frac{1}{4 \pi \varepsilon_{0}} \int_{-h / 2}^{h / 2} d z^{\prime \prime} G_{m j}\left(\vec{k}, z^{\prime}, z^{\prime \prime}\right) \chi_{j l}(\omega, z) e^{i q\left(z-z^{\prime}\right)}\right]\right\}=0 .
\end{aligned}
$$

In this work, we numerically analyze the dependence of the normalized dissipative function $I=Q / I_{0}$ on the frequency and angle of incidence for the probing radiation. Eq. (13) is the main expression that was used in this work for numerical calculations.

\section{Numerical results and discussions}

Using the analytical approach developed in Sec. 4, here we analyze numerically the characteristic features of light absorption by thin inhomogeneous semiconductor film. To analyze numerically the features of the process of light absorption by the inhomogeneous semiconductor film, we have chosen the following parameters of the film: thickness of the film $h=40 \mathrm{~nm}$, concentration of electrons in the undoped film $n_{0}=2 \cdot 10^{18} \mathrm{~cm}^{-3}$, dose of implanted ions $N_{i}=1.2 \cdot 10^{14} \mathrm{~cm}^{-2}$, and dielectric permeability of GaAs $\varepsilon=13.1$. Study of light absorption by the thin semiconductor film with inhomogeneous distribution of compensating impurities shows that the absorption profile has interesting characteristic properties. First of all, it is necessary to note that, in contrast to the well-known electric dipole approximation where absorption at the normal light incidence is absent $[1,24]$, the present calculations show nonzero absorption at the same condition. This fact is connected with the use of more general model that does not base on the electric dipole approximation and localfield effects. As it follows from the numerical results (see, Figs 4-7), the main characteristic properties of absorption processes is the presence of one or two peaks in absorption profile determined by plasma oscillations. Indeed, the continuous distribution of the carriers along the thickness of the film should lead to rather wide absorption spectrum caused by interaction between the light and electron plasmas in semiconductor material of the film. Moreover, the broadening of the impurity distribution leads to a shift of the peaks, which corresponds to the plasma oscillations, to a lowfrequency region. Another interesting feature of the absorption processes is the growth of an additional plasma peak at a lower frequency relatively to the main plasmon peak. This low-frequency peak exhibits tendency to the shift to the low-frequency region, when the impurity distribution becomes broader. It should be noted that the low-frequency peak shifts much faster than the main one. The results of the numerical calculations are shown in Figs 4-7. The calculations demonstrate that continuous distribution of the impurities, which mean that plasma frequency is distributed continuously along the thickness of the film, leads to the increase of absorbing profile with one or several peaks. The characteristic feature of absorption processes is the dependence of absorption spectra on the width of the layer doped with impurities and on the depth of their occurrence. The dependence of the absorption intensity on the incident light frequency and 


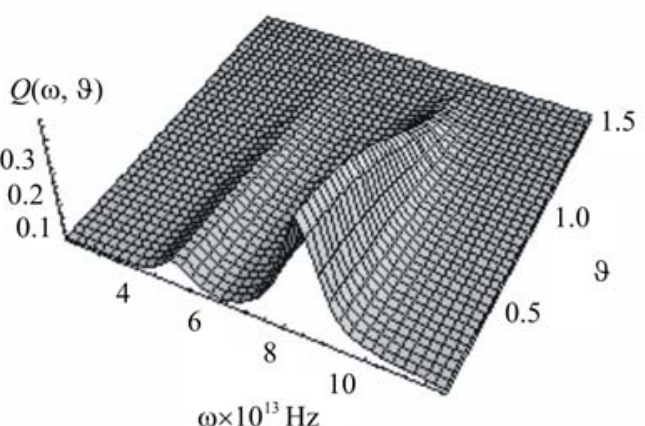

$a$

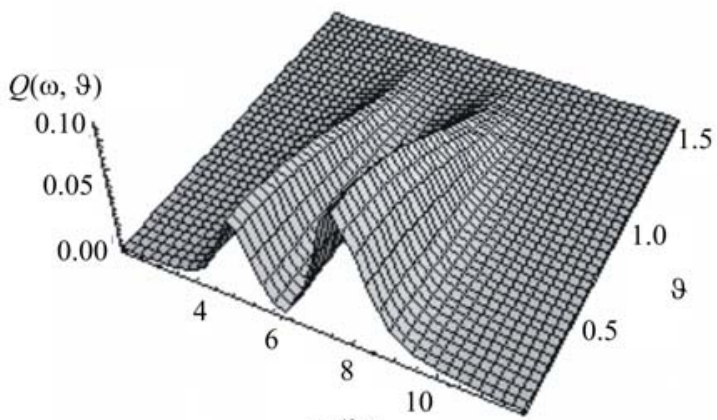

$\omega \times 10^{13} \mathrm{~Hz}$

$b$

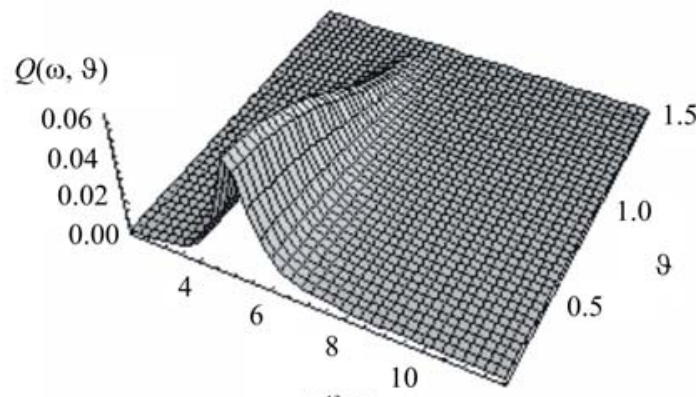

$\omega \times 10^{13} \mathrm{~Hz}$

$c$

Fig. 4. Absorption profiles for the thin film $(h=40 \mathrm{~nm})$ of $p$-polarized light in the cases of narrow, intermediate, and wide impurity distributions, respectively. The distribution located in the middle of the film $R_{p}=0.5 \mathrm{~h}$. a) $\Delta R_{p}=0.01 \mathrm{~h}$,

b) $\Delta R_{p}=0.06 h$, c) $\Delta R_{p}=0.3 h$.

angle of light incidence is shown in Fig. 4. This figure demonstrates the behavior of the absorption spectrum in the case of different widths of impurity distribution: narrow (a), intermediate (b), and wide (c). The broadening of distribution leads to diminution of the absorption intensity and to splitting the plasmon peak (Fig. 4b). The plasmon peak disappears in the case of a very wide distribution of impurities (see Fig. 4c) that confirm total compensation of the electrons in the film by ion impurities. Fig. 5 exhibits the profiles of the absorption intensity dependence on the frequency with the fixed angle of incidence $\vartheta=11^{\circ} 46^{\prime}$, which helps to understand the behavior of $3 \mathrm{D}$-figures in Fig. 4 . The comparison of absorption spectra for different positions of the distribution of impurities is demonstrated in Fig. 5 for the case of maximum of impurity distribution located in the middle of the film, namely, $R_{p}=0.5 \mathrm{~h}(\mathrm{a})$, and for the case of shifted distribution $R_{p}=0.2 h$ (b).

The absorption spectra with sufficiently wide distribution of implanted ions are shown in Fig. 6. The evolution of splitting and shifting the plasmon peak of absorption profile is also demonstrated in Fig. 6. First of all, the splitting of the plasmon peak, when the distribution becomes wider, arises. The splitting increases, when broadening of impurity profile continues, and both low-frequency and high-frequency peaks shift to the longwave region. Moreover, the shifting of the low-frequency peak is appreciably faster than shifting of the high-frequency plasmon peak.
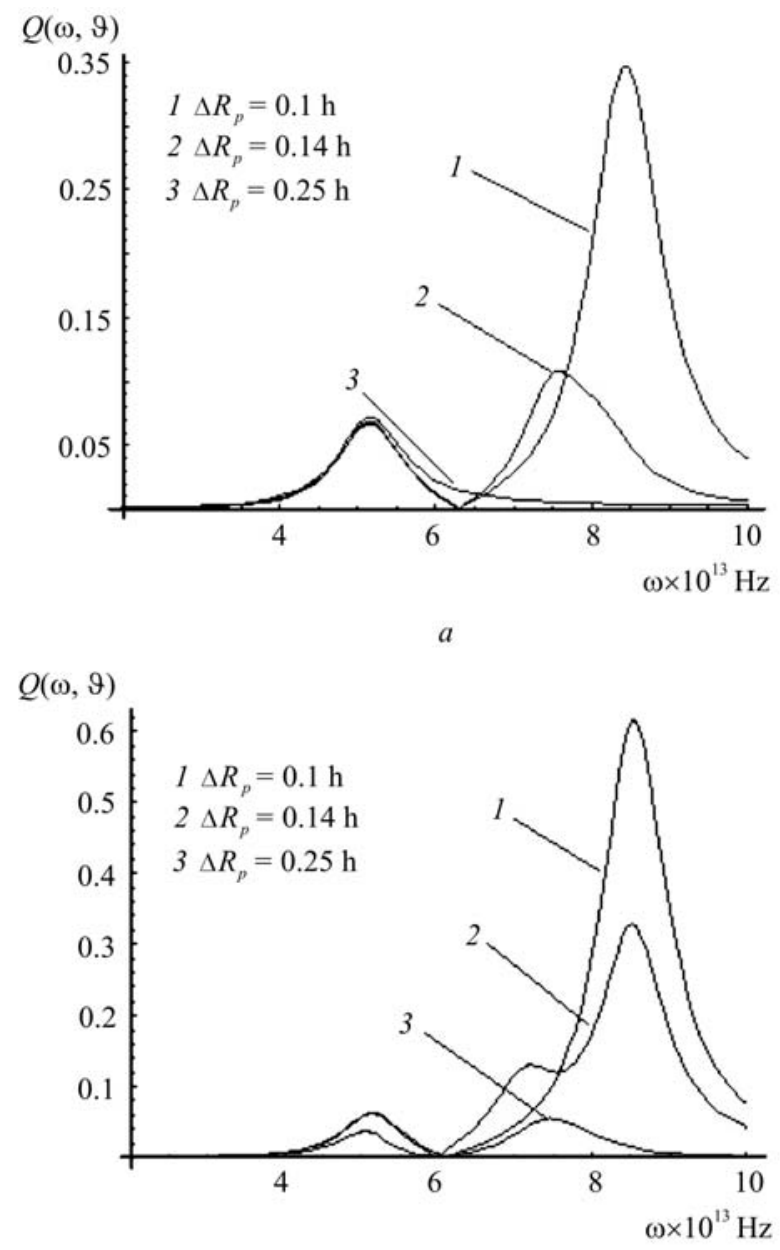

$b$

Fig. 5. Dependences of the absorption energy on the frequency of external radiation for the cases of narrow, intermediate, and wide distributions. The angle of incidence

$\vartheta=11^{\circ} 46^{\prime}$. a) The distribution centered in the middle of the film $\left.R_{p}=0.5 h . \mathrm{b}\right)$ The center of the distribution is shifted from the middle of the film $R_{p}=0.2 \mathrm{~h}$. 

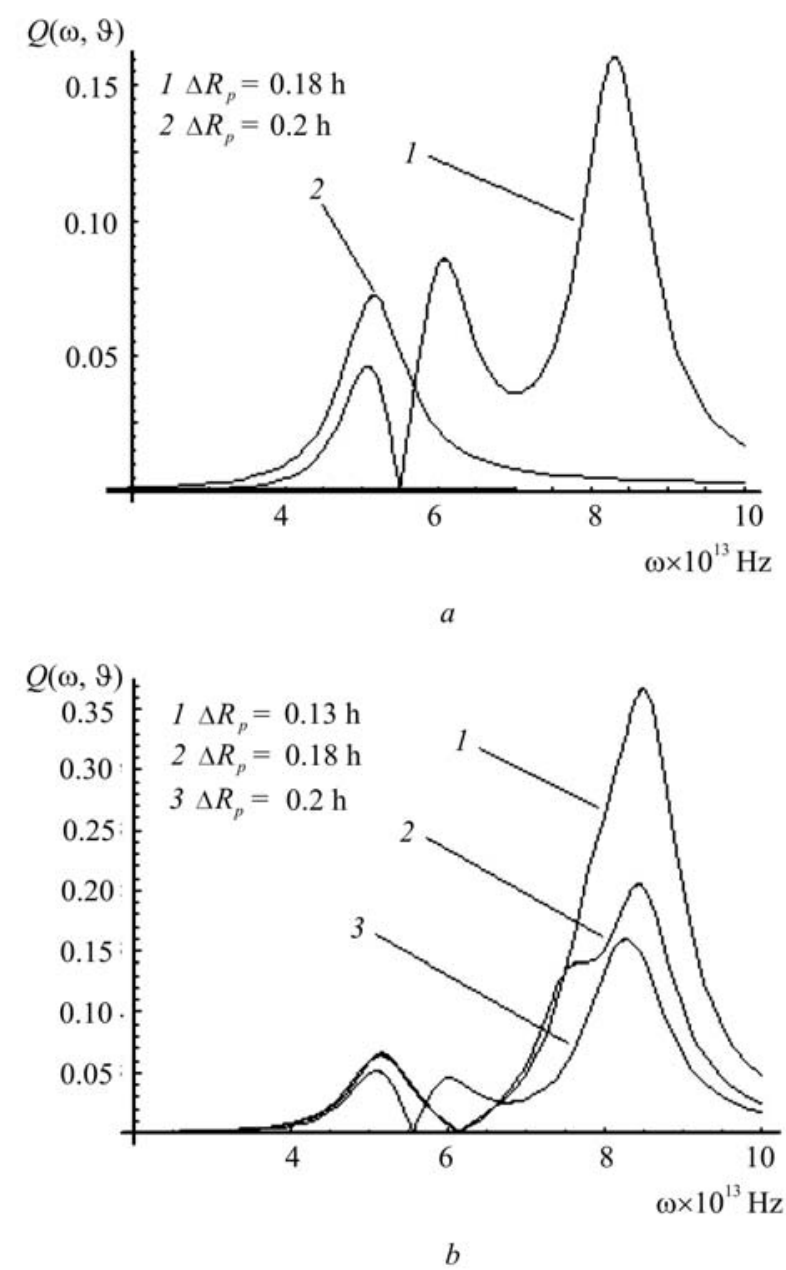

Fig. 6. The absorption profiles of the p-polarized wave in the case of wide distributions with regard to "distribution tails". The angle of incidence $\vartheta=11^{\circ} 46^{\prime}$. a) The distribution is centered in the middle of the film $\left.R_{p}=0.5 \mathrm{~h} . \mathrm{b}\right)$ The center of distribution is shifted from the middle of the film $R_{p}=0.2 h$.

These characteristic features can be explained by the following speculations. The film was characterized by inhomogeneous distribution of plasma oscillations with different frequencies. Different plasmons are characterized by different phase volumes. It means that contribution into interaction "plasma oscillations-light" at different frequencies will be different. To clarify this thought, let us consider Fig. 8 where the different widths of impurity distributions along the film thickness are shown. According to general properties of solid state plasma, the response function that was connected to the total induced charge with the total electric potential is proportional to the correlation function of the "charge density - charge density" type (see, for example Ref. [23]). Then the interactions between light and plasma subsystems of the film is weaker, the electron concentration is lower. Let us consider the narrow distribution of compensating impurity (Fig. 7a). It is convenient to divide the distribution profile into three regions, where the points "a" and "b" are the inflections of the Gauss curve. As a result, one can contend that the compensation is practically absent in the regions 1 and 3 . It can be supposed that the plasma oscillations in these regions will be characterized by the frequency $\omega_{p}^{1,3}$ (defined by the electron concentration corresponding to points in the surface region of the film) that is approximately equal to the plasma frequency of undoped semiconductor $\omega_{p}^{\max }$ that is connected with the electron concentration $n_{0}$ of uncompensated material via the expression

$\left(\omega_{p}^{\max }\right)^{2}=\frac{q^{2} n_{0}}{m \varepsilon \varepsilon_{0}}$.

In the region 2, distribution of impurities is evident. The reason is that in the narrow region the impurity concentration is quickly changed, the region 2 of impurity distribution can be characterized by any frequency $\omega_{p}^{2}$ that can be selected approximately as a frequency corresponding to the most strong interaction between light and plasma subsystems. It is clear that $\omega_{p}^{2}$ will be not essentially differ from $\omega_{p}^{1,3}$. Moreover, the contribution into the absorption processes of the region 2 is much less than those of regions 1 and 3 , or $I\left(\omega_{p}^{2}\right)<<I\left(\omega_{p}^{1,3}\right)$. It means that absorption profile corresponding to light-plasma interaction is determined only by the oscillations with the frequency $\omega_{p}^{1,3}$. Then, the total absorption profile should have two peaks - one of them (lower frequency) corresponds to light-lattice subsystem interaction, and the second (higher frequency) corresponds to light-plasma interaction (Fig. 4a and Fig. 5, curve 1).

If the profile of impurity distribution has an intermediate width (Fig. 7b), the concentration profile varies more smoothly. The inflections (pointed as $a^{\prime}$ and $\left.b^{\prime}\right)$ and boundaries of the regions $\left(1^{\prime}, 2^{\prime}, 3^{\prime}\right)$ vary their positions. The $1^{\prime}, 3^{\prime}$ regions become narrower. It means that the region where plasmons with the frequency $\omega_{p}^{\max }$ play the main role become narrower. The electron concentration in these regions slightly decreases because the "distribution tails" are involved outside the film. This circumstance leads to weak decreasing interaction between light and plasma oscillations at frequencies about $\omega_{p}^{1^{\prime}, 3^{\prime}}$. It means that the peak of absorption profile characterized by frequency $\omega_{p}^{1^{1}, 3^{\prime}}$ becomes lower. The central frequency of the peak remains equal to $\omega_{p}^{1^{\prime}, 3^{\prime}} \approx \omega_{p}^{\max }$ because the thickness of regions 1', 3' keeps rather wide. A more interesting behavior of plasma oscillations is displayed in the region $2^{\prime}$. This region is characterized by lower frequencies as in $1^{\prime}, 3^{\prime}$ regions. Then, $\omega_{p}^{\prime 2}<\omega_{p}^{2}$, and the possibility of 


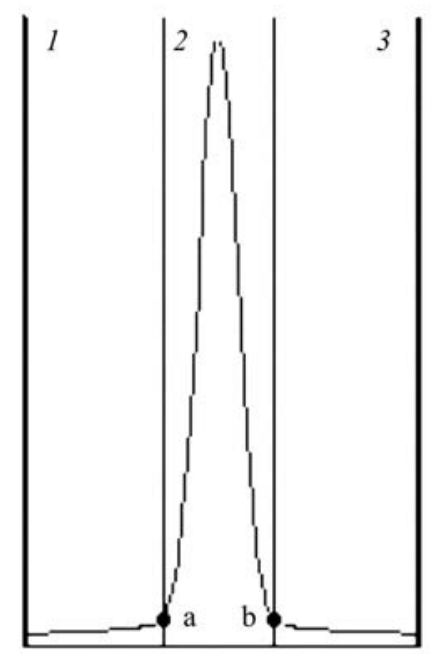

$a$

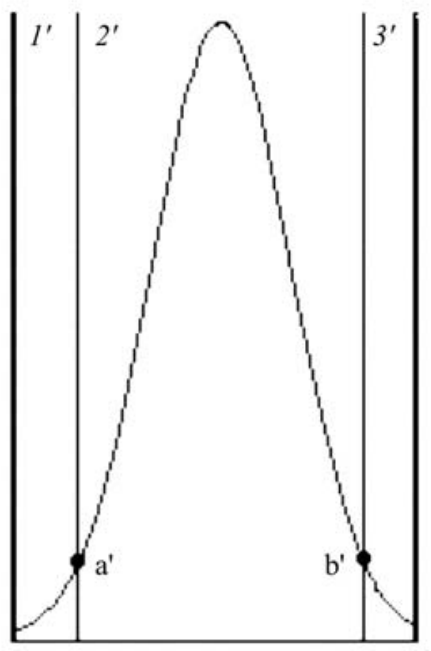

b

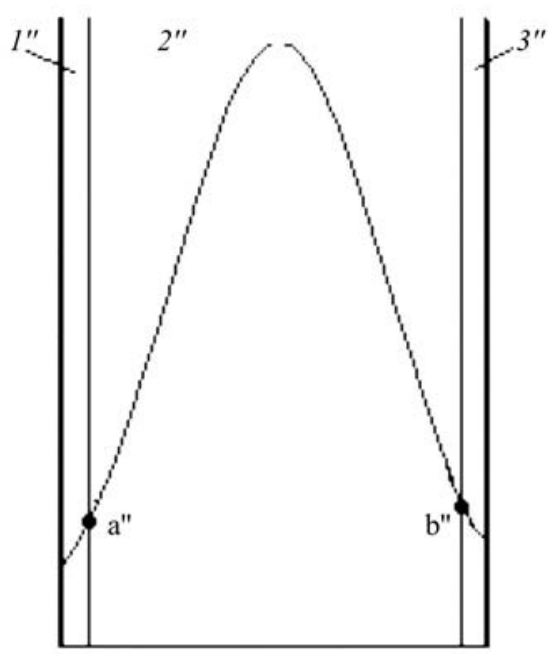

c

Fig. 7. Three cases of location of "distribution tails” inside the film.

the additional peak growth appears. This situation is shown in Figs $4 \mathrm{~b}$ and 5 (curve 2) where the plasmon peak splits. Further broadening of the distribution profile (Fig. 7c) leads to enhancement of effect of plasmon peak splitting in the absorption profile. Moreover, the characteristic plasmon frequency for the regions $1^{\prime \prime}$ and $3^{\prime \prime}$ shifts to the low-frequency region, because compensation processes become to play role in the regions near film boundaries diminishing the electron concentration in the regions. Then, the inequality becomes valid $\omega_{p}^{1^{\prime \prime}, 3^{\prime \prime}}<\omega_{p}^{\max }$. This fact leads to decreasing the "light-electron plasma" interactions. Then the intensity of the main plasmon peak in absorption profile decreases. One should note that the shift velocity of the peaks in the regions 1 and 3 is smaller than that of the peaks caused by plasmons in the region 2. This is because of distribution function profile after inflection (in the tail regions 1 and 3 ) has more slow behavior than in the middle of the region 2 .

\section{Conclusions}

In this work, we used the local field method based on the formally exact solution of the Lipmann-Schwinger equation to analyze the light absorption processes by a thin semiconductor film with an inhomogeneous distribution of the compensating impurity. Then, the electrodynamical properties of the thin film were calculated in the frames of the self-consistent approach. As a result, the effective susceptibility of thin film was calculated analytically. To calculate the absorption spectra for a strongly inhomogeneous system, we used the dissipation function approach. This calculation showed that the dissipative processes are not directly determined by the imaginary part of the effective susceptibility of the system (linear response to the external field).

The absorption spectra were calculated numerically. The spectra exhibited some unexpected features: first, a splitting of the plasmon peak under the conditions of sufficiently wide impurity distribution; second, different shift velocities for each plasmon peak when the peaks shift into the low-frequency range with the impurity distribution widening. The characteristic behavior of the absorption profile consisting of the absorption peaks connected with plasmon oscillations, when the electron concentration is distributed continuously, was discussed.

It should be mentioned that results, obtained in this work, are qualitatively confirmed by experimental data demonstrated in Ref. [21].

\section{References}

1. V. Mitin, V. Kochelap, M. Stroscio, Quantum heterostructures. Microelectronics and optoelectronics. University Press, Cambridge (1998).

2. J. Davies, The physics of low-dimensional semiconductors. Cambridge, University Press (1998).

3. L.K. Chopra, Thin film phenomena. Robert E. Krieger Publish. Inc. Malabar, Florida, (1985).

4. M.L.H. Lahlaouti, A. Akjouj, B. Djafari-Rouhani, and L. Dobrzynski, Resonant and localized electromagnetic modes in finite superlattices // Phys. Rev. B 61, p. 2059-2064 (2000).

5. S.R. Forest, Ultra-thin organic films grown by organic molecular beam deposition and related techniques // Chem. Rev. 97, p. 1793-1896 (1997).

6. J.M. Tour, Molecular electronics. Commercial insights, chemistry, devices, architecture and programming. World Scientific, London (2003). 
7. K. Ploog, Processing light signals: Applications of MBE-grown quantum wells and superlattices to laser and other photonic devices // Physics and Technology, 19, p.196-205 (1988).

8. V.B. Gorfinkel, S. Luryi, Dual modulation of semiconductor lasers // Proc. SPIE 2114, p. 204-209 (1994).

9. M. Hennini, M. Leadbeater, E.S. Alves, L. Eaves and O.H. Hughes, Ballistic transport in resonant tunneling divices with wide quantum wells // $J$. Phys.: Condens. Matter 1, p.3025-3030 (1989).

10. C, Nylander, B. Liedberg, T. Lind // Sensors and Actuators 3 p. 79-88 (1982).

11. V. Chegel, Yu. Shirshov, S. Avilov, M. Demchenko, M. Mustafaev, A novel aldehyde dextran sulfonate matrix for affinity biosensors // J. Biochem. Biophys. Methods 50, p. 201-216 (2002).

12. O. Keller, Local fields in the electrodynamics of mesoscopic media // Phys. Repts 268, p. 85-262 (1996).

13. C. Girard, C. Joachim, S. Gauthier, The physics of the near-field // Repts Prog. Phys. 63, p. 893-938 (2000).

14. B.I. Khudik, V.Z. Lozovski, A.F. Zhuravlev, Optical theorem in ultra-thin film electrodynamics // Physica status solidi (b) 151, p.111-119 (1989).

15. B.I. Khudik, V.Z. Lozovski, I.V. Nazarenko-Baryakhtar, Macroscopic electrodynamics of ultra-thin films // Physica status solidi (b) 163, p. 167-177 (1989).
16. B.I. Khudik, V.Z. Lozovski, A.F. Zhuravlev, About boundary conditions in ultra-thin film electrodynamics // Ukr. Fiz. Zhurn. 34, p. 62-64 (1989) (in Russian).

17. O. Keller, Sheet model description of the linear optical response of quantum wells // J. Opt. Soc. Amer. B 12, p.987-996 (1995).

18. S. Bozhevolnyi, V. Lozovski, Self-consistent model for second-harmonic near-field microscopy // Phys. Rev. B 61, p.1139-1150 (2000).

19. V. Lozovski, Yu. Nazarok， S. Bozhevolnyi， Nearfield imaging of pyramid-like nanoparticles at a surface // Physica E 11, p.323-331 (2001).

20. H. Rissel, I. Ruge, Ion implantation. Springer, Berlin (1980).

21. E. Venger et al. // Fiz. Techn. Poluprovodnikov 26 p. 352-357(1992)(in Russian).

22. L. Baraban, V. Lozovski, Reflection and absorption of the light by thin semiconductor film // Optika $i$ Spektroskopiya 97, p.810-816 (2004) (in Russian).

23. P.M. Platzman, P.A. Wolff, Waves and interactions in solid state plasmas. Academic Press, New York, London (1973).

24. M. Helm, in: Intersubband transitions in quantum wells, ed. by H.C. Liu and F. Capasso. Academic Press, San Diego (2000). 